calprotectin and MPO in plasma showed statistically significant differences in related to neoadjuvant treatment. (Figure 2).

Conclusion* The observed correlation between the values of the different markers and neutrophils' levels suggests their validity as biomarkers of NETosis. For the first time, we describe an increase in NETosis markers in ascites of advanced HGSOC, which could show the contribution of NETosis in the progression of HGSOC in the pelvic microenvironment. Furthermore, cfDNA in plasma could represent a minimally invasive diagnostic biomarker for HGSOC that could reduce the pre-surgical rate of false positives.

\section{TOTAL POLYUNSATURATED FATTY ACID LEVEL IN ADIPOSE TISSUE AS AN INDEPENDENT PREDICTOR OF RECURRENCE-FREE SURVIVAL IN WOMEN WITH OVARIAN CANCER}

${ }^{1} \mathrm{~L}$ Ouldamer*, 'S Hélène, ${ }^{2} \mathrm{D}$ Adeline, ${ }^{2} \mathrm{~S}$ Stephane, ${ }^{2} \mathrm{D}$ Jean-François, ${ }^{1} \mathrm{G}$ Caroline. ${ }^{1} \mathrm{Ch} u$ Bretonneau Tours, Tours, France; ${ }^{2}$ Nutrition, Growth and Cancer, France

\subsection{6/ijgc-2021-ESG0.605}

Introduction/Background* Prognostic factors for epithelial ovarian cancers (EOCs) are in particular clinical factors such as pathology staging at diagnosis (FIGO stages), genetic mutation or histological phenotypes. In the present study, we explored whether fatty acid composition of adipose tissue, may be associated with recurrence-free survival in EOC.

Methodology Forty-six women with epithelial ovarian cancers and 6 with borderline ovarian tumors were included between March 2017 and January 2020 in this prospective study in Tours university teaching hospital (central France). Adipose tissue specimens from four abdominal locations (superficial and deep subcutaneous, visceral epiploic and omental) were collected during surgery or exploratory laparoscopy. A fatty acid profile of adipose tissue triglycerides was established by gas chromatography. We assessed differences associated with disease recurrence.

Result(s)* The content of long-chain saturated fatty acids (SFAs) was increased and that of long-chain polyunsaturated fatty acids (PUFAs) decreased in deep versus superficial subcutaneous adipose tissue in EOC patients. Nevertheless, the content of total SFAs was 28\%, monounsaturated fatty acids (MUFAs) 55\%, PUFAs n-6 11.5\% and PUFAs n-3 about 1.3\% whatever the adipose tissue. For EOC patients, median followup was 15 months. FIGO stage, tumor residue after surgery and body mass index, were clinical predictors of recurrencefree survival (RFS). EOC patients were separated into two groups by median fatty acid content. Content of total PUFAs $(n-6+n-3)$, whatever the adipose tissue, was associated with RFS. RFS was about 2 times longer in EOC patients with high versus low total PUFA content (median survival: 12 vs 27 months, $\mathrm{p}=0.01$ to $<0.0001$ according to the tissue).

Conclusion* Content of total PUFAs $(n-6+n-3)$ in abdominal adipose tissue (visceral and subcutaneous) are new prognostic factors in EOC. The origin of PUFAs depletion needs to be explored, to design a new therapeutic strategy for these patients

\section{Trophoblastic diseases}

\section{STEVEN JOHNSON SYNDROME (SJS) COMPLICATING METHOTREXATE (MTX) THERAPY FOR CHORIOCARCINOMA. A CASE REPORT AND REVIEW OF THE LITERATURE}

${ }^{1} \mathrm{E}$ Brewster, ${ }^{2} \mathrm{SS}$ Superville*. 'Morehouse School of Medicine, Obstetrics and Gynaecology, USA; ${ }^{2}$ Port of Spain General Hospital, Surgery, Trinidad and Tobago

\subsection{6/ijgc-2021-ESG0.606}

Introduction/Background* Successful management of choriocarcinoma with single-agent methotrexate is documented since 1956. Methotrexate multiday-regimen remains the typical first-line therapy in low-risk Gestational Trophoblastic Neoplasia (GTN). GTN is very sensitive to chemotherapy but evidence on serious adverse events is of low certainty. Stevens-Johnson Syndrome (SJS) is a severe cutaneous adverse reaction with a high mortality rate. This to the best of our knowledge is the second case in the medical literature of SJS following the use of intermediate-dose methotrexate $(>50$ to $<500 \mathrm{mg} / \mathrm{m} 2$ ).

Methodology 33-year-old woman noted with rising serum Beta-Human-Chorionic-Gonadotropin (BHCG) level two months after Caesarean delivery of term singleton gestation. BHCG rose from 5160 in June to $68,300 \mathrm{mlU} / \mathrm{ml}$ in July. Past medical history significant for genital herpes-simplexvirus infection. Sonogram showed a vascular mass within the endometrial cavity. Subsequently underwent suction dilatation and curettage with pathological diagnosis of choriocarcinoma .Treated as an outpatient with methotrexate $1 \mathrm{mg} /$ $\mathrm{kg}$ (66mg total dose) by subcutaneous injection daily for five days.

Patient presented with fever, pruritic external genital vesicles and dysphagia two days after fifth methotrexate dose. Vitals were stable. A diffused erythematous maculovesicular rash was noted on face and chest with painful white plaques on oropharyngeal mucosa. She was admitted with a presumptive diagnosis of oropharyngeal thrush, disseminated herpes infection and stomatitis.

Result(s)* Despite antiviral therapy, skin lesions progressed to upper back and vaginal mucosa. Serological and direct fluorescent antibody tests for HSV1, HSV2 and varicella virus were negative. Dermatology consult reported skin biopsied histopathological findings most compatible with SJS. The following week dysphagia resolved and desquamated lesions were resolving. At discharge BHCG level decreased to 39.7 $\mathrm{mIU} / \mathrm{ml}$.

Conclusion* Our case highlights the diagnostic dilemma of a life threatening adverse drug reaction to methotrexate therapy for low risk GTN. Despite being rare, we believe that SJS should be considered in patients presenting with adverse reactions to methotrexate within or outside guideline regimens. Early diagnosis and management of SJS can limit mortality and sequelae and in the case of GTN may involve the selection of an alternative single agent chemotherapy if further treatment is warranted. 